\title{
Scolia
}

Revue de linguistique

$31 \mid 2017$

Le proverbe

\section{Le fonctionnement du temps et de l'aspect dans la gnomicité / généricité des proverbes}

Jean-Claude Anscombre

\section{(2) OpenEdition}

\section{Journals}

Édition électronique

URL : http://journals.openedition.org/scolia/409

DOI : $10.4000 /$ scolia.409

ISSN : 2677-4224

Éditeur

Presses universitaires de Strasbourg

Édition imprimée

Date de publication : 20 juin 2017

Pagination : 11-37

ISBN : 978-2-86820-964-1

ISSN : $1253-9708$

Référence électronique

Jean-Claude Anscombre, « Le fonctionnement du temps et de l'aspect dans la gnomicité / généricité des proverbes », Scolia [En ligne], 31 | 2017, mis en ligne le 09 avril 2019, consulté le 18 mai 2019.

URL : http://journals.openedition.org/scolia/409; DOI : 10.4000/scolia.409 


\title{
Le fonctionnement du temps et de l'aspect dans la gnomicité/généricité des proverbes ${ }^{1}$
}

\author{
Jean-Claude Anscombre \\ CNRS/LDI \\ jcanscombre@free.fr
}

\section{Introduction}

Un des grands mérites de la linguistique contemporaine est d'avoir sorti la parémiologie du cabinet des objets folkloriques où elle végétait depuis un certain temps, et de lui avoir restitué son titre «d'objet linguistique ». Il faut donc saluer comme il se doit les travaux novateurs (et rénovateurs) d'un certain nombre de parémiologues, tant sur le plan de la diachronie que sur celui de la synchronie, et dans divers compartiments du phénomène parémique: citons au hasard (et parmi beaucoup d'autres) les nombreuses contributions de Mirella Conenna, Georges Kleiber, Julia Sevilla et Irène Tamba pour la synchronie, Alexandra Oddo, Bernard Darbord, Elena Llamas Pombo et Amalia Rodríguez Somolinos pour la diachronie.

Une fois accordé ce satisfecit à la profession de parémiologue moderne, force est de reconnaitre qu'elle n'est pas de tout repos. D'une part, il convient d'expulser définitivement un certain nombre de vulgates qui semblent avoir la vie dure. D'autre part, il faut les remplacer par un apparat linguistique opératoire, ce qui n'est pas chose

1 Tous mes remerciements à I. Tamba (EHESS-CRLAO), S. Deloor (Univ. de CergyPontoise) et S. Gómez-Jordana (Univ. Complutense de Madrid) pour les nombreuses discussions sur les points évoqués dans ce travail. 
facile. Comme beaucoup d'objets linguistiques qui nous sont familiers, l'objet parémie a un caractère de fausse évidence vite détrompé par la difficulté à en circonscrire la nature et le fonctionnement.

Je rappellerai dans un premier temps l'essentiel de mes positions sur la parémiologie, à quel type de questionnement elles répondent et à quelles autres positions elles s'opposent. En particulier à l'hypothèse assez répandue des proverbes comme formes, et formes figées. Dans le cadre de l'hypothèse principale que je soutiens (nous y reviendrons) - les parémies sont l'association spécifique d'une forme et d'un sens - je tenterai de résoudre le problème de la mise en évidence d'un des caractères fondamentaux des parémies: leur généricité/gnomicité. Il y a en effet un accord général sur le fait que les parémies dénotent des sortes de vérités universelles: elles sont gnomiques, et des chercheurs comme Georges Kleiber et moi-même voyons cette gnomicité comme proche de la généricité d'énoncés typifiants a priori, ainsi Les chats chassent les souris ou encore Les incendies ravagent les forêts. Appuyer cette proposition (qui semble raisonnable) sur des critères opératoires ne va pas de soi: le lien entre forme et sens qui caractérise une parémie est très particulier ${ }^{2}$.

\section{Les phrases parémiques}

Une fois le phénomène parémique réintégré dans le corps des phénomènes linguistiques, il convient de lui donner un statut linguistique, i.e. de le constituer en tant que catégorie linguistique. Il faut donc imaginer des critères aptes à le cerner, afin d'éviter au maximum le recours à des mots de la langue comme «forme brève» ou «maxime» dont j'ai démontré ailleurs le caractère totalement illusoire et non opératoire (Anscombre, 2000, 2007, 2010). Je me placerai à l'intérieur du cadre théorique auquel j'ai consacré plusieurs publications (cf. par exemple Anscombre, 2012a). L'examen de plusieurs milliers d'exemples, dans plusieurs langues et dans des corpus variés, m'a permis de relever des propriétés stables, qui m’ont conduit à proposer l'approche et la classification suivantes (Anscombre, 2008):

2 Sur ce point, cf. Tamba (2014). Pour éviter d'alourdir le texte, voire le rendre illisible, les traductions des proverbes anglais, espagnols et portugais se trouvent en annexe à la fin. 


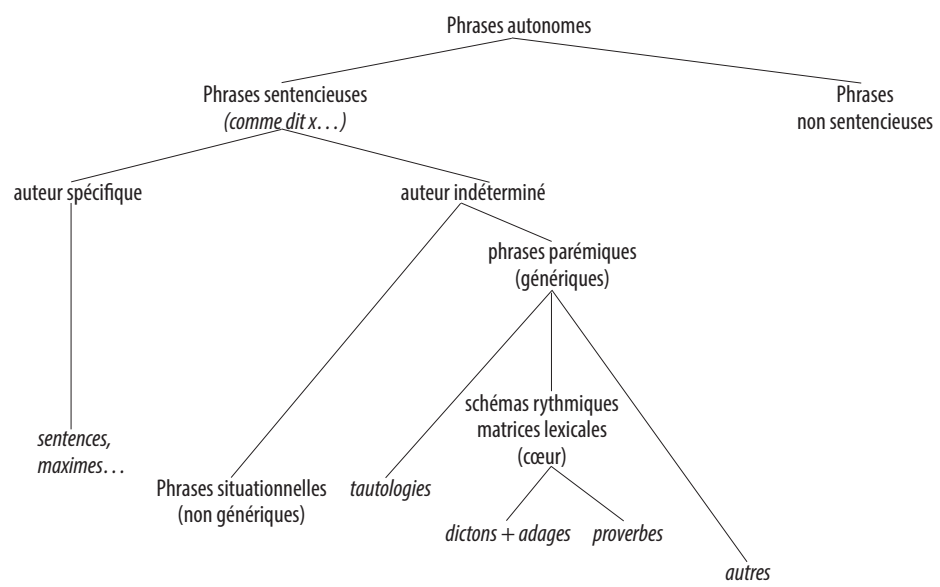

Ce schéma appelle un certain nombre de commentaires:

a) L'appellation phrase autonome est un raccourci commode pour "phrase dont l'énoncé est autonome», (Anscombre, 2016a). Pour ce qui est de la notion de phrase et les problèmes qu'elle soulève - et qui sont loin d'être résolus - je suivrai volontiers l'approche de Martin (2016), sur la phrase comme contour intonatif spécifique, en particulier en ce qui concerne le phénomène parémique. Elle recoupe l'étude de Llamas Pombo (2016), sur les signes graphiques de la ponctuation forte comme marque de l'autonomie phrastique des proverbes. Le phénomène parémique dans son ensemble repose tout d'abord sur un phénomène de médiativité ${ }^{3}$. La catégorie la plus générale, celle des phrases sentencieuses, se caractérise par l'existence d'une source bien précise pour chacune d'entre elles. Cette source est ou bien explicite (ce qui est presque toujours le cas lorsqu'il s'agit d'une entité particulière), ou bien explicitable sous la forme de comme dit $x, x$ étant bien entendu la source de la phrase (ou forme) sentencieuse. Les phrases parémiques se caractérisent par un $x=$ on ou équivalent. La tournure la plus fréquente est comme

3 Le terme médiativité est l'équivalent de l'anglais evidentiality: il désigne l'ensemble des procédés linguistiques spécifiant, pour un énoncé donné, la source d'une information, le mode d'accès à cette information et la position éventuelle du locuteur par rapport à ces paramètres. 
on dit pour le français, como se dice o dicen pour l'espagnol, as they say pour l'anglais, etc. Je n'ai pour l'instant pas pu trouver de langue où un tel introducteur de médiativité n'existe pas. Par ailleurs, comme on dit n'est pas une simple cheville et possède des propriétés linguistiques tout à fait spécifiques, et reliées à la notion de communauté linguistique anonyme (cf. sur ces points Anscombre, 2006, 2010, entre autres) 4 .

b) La notion de cœur d'une famille parémique renvoie au fait que les éléments prototypiques de la catégorie des parémies correspondent à des matrices lexicales et à des schémas rythmiques, notions sur lesquelles je reviendrai plus avant.

c) Les «sorties» de cette classification correspondent grosso modo aux entités en italique (pour indiquer qu'il s'agit des notions habituelles de proverbe, maxime, etc.) ${ }^{5}$.

d) Si on se borne aux phrases sentencieuses à auteur indéterminé, on remarque qu'elles se séparent en deux sous-classes, les phrases situationnelles et les phrases parémiques. En voici quelques exemplaires:

1) En cuanto a vuestro pleito por los ocho mil cuatrocientos reales del duque de Osuna, ya sabéis que las cosas de palacio van despacio (Pérez-Reverte, Limpieza de sangre. (Las aventuras del capitán Alatriste II), 2003)

2) Ya sé que hubo un tiempo en que no nos llevábamos bien usted y yo - añadió -, pero eso ha pasado a la historia. Acepte mi invitación y pelillos a la mar. (Mendoza, La verdad sobre el caso Savolta, 1975)

3) Son nom venait brusquement de revenir à la mémoire d'Adamsberg... Pour le prénom, rien à faire, à l'impossible nul n'est tenu. (Vargas, Pars vite et reviens tard, 2001)

4) - J'ai tenté d'établir le profil psychologique du meurtrier avec un collègue criminologue. - Je croyais que vous n'étiez plus en charge $d u$ dossier... - Eh, Mackensie (à qui on vient de retirer le dossier),

4 Ce qui montre que, contrairement à une vulgate largement répandue, le proverbe n'est pas une banale citation.

5 Il n'est en effet nullement assuré qu'on puisse définir une catégorie comme dicton par des critères linguistiques stables. Les mots de la langue ne sont pas des concepts opératoires. 
c'est l'Hôpital qui se fout de la Charité. (Loevenbruck, Le rasoir d'Ockham, 2008)

5) As the Scots say, and they should know, many a mickle mak's a muckle. (Huntly Express, 1940, 19 janvier, 3).

Le problème de l'identification des phrases parémiques se ramène en fin de compte à une double identification:

i) montrer qu'on se trouve dans la catégorie des phrases sentencieuses (au sens ci-dessus). C'est le point le moins ardu, étant donné que beaucoup de textes comportent des introducteurs du type de comme on dit ou équivalents, comme le montre l'échantillonnage suivant:

6) Au premier abord, la neige ne paraît pas constituer un danger pour l'environnement. Mais comme le dit le vieil adage "Méfiezvous de l'eau qui dort!». En effet, au printemps, lorsque la neige fond, elle libère des polluants. (Le journal du CNRS, $\mathrm{n}^{\circ} 172,2004$ )

7) S'agissait-il d'un complot? Mais trop d'eau avait passé sous les ponts, comme on dit, pour qu'elle cherchât à se venger après toutes ces années. (Bouillier, L'Invité mystère, 2004)

8) De tal palo, tal astilla, suele decirse. Muchas veces la descendencia no continúa la tradición familiar, aunque en el caso del grupo Dikers uno de sus integrantes sí ha seguido los pasos del padre. Con sólo 17 años, Iker Piedrafita, hijo del guitarrista de Barricada Alfredo Piedrafita, toca la guitarra, canta y compone en un cuarteto de estudiantes navarros. (El País, 8/5/99)

9) La política partidaria se rige por ese viejo principio que dice que la confianza da asco, precepto que guía también las relaciones familiares o de amistad, que suelen ser siempre bastante más tempestuosas que las que se mantienen con desconocidos. (El País, 19/2/99)

ii) Montrer qu'on se trouve en présence d'une phrase parémique et non d'une phrase situationnelle, ce qui revient à montrer la généricité/gnomicité de la phrase sentencieuse considérée. Le problème est que si certains cas prototypiques semblent incontestables (sous réserve d'inventaire...), ainsi:

10) Chacun voit midi à sa porte.

11) Il ne faut jamais remettre au lendemain ce qu'on peut faire le jour même.

12) Cría cuervos, y te sacarán los ojos. 
13) Cuando el río suena, agua lleva.

14) Quem tudo quer, tudo perde.

15) Mais vale um pássaro na mão que dois voando

16) A friend in need is a friend indeed.

17) A rolling stone gathers no moss.

D'autres cas semblent beaucoup moins évidents, ainsi :

18) C'est l'hôpital qui se moque de la charité.

19) La confianza da asco.

Nous commencerons par passer en revue certains indices qui peuvent indiquer la généricité, avant de proposer une analyse reposant sur la nature sémantique du proverbe, en particulier le temps et l'aspect.

\section{Divers indices de généricité / gnomicité}

\subsection{Les indices diachroniques}

Une idée qui vient assez naturellement à l'esprit est de chercher dans l'histoire d'une forme sentencieuse des indices de sa généricité. Le raisonnement repose sur l'idée qu'une forme sentencieuse qui a été générique à une certaine époque et qui s'est transmise jusqu'à l'époque actuelle a de bonnes chances d'être générique. Une forme forte de cette hypothèse est que ce qui a été proverbe un jour l'est pour toujours, et peut être réactivé à n'importe quel moment. C'est la vulgate bien connue de la transmission fidèle des formes sentencieuses de génération en génération, et elle est indéfendable. Les énoncés suivants ont été des proverbes:

20) Cheval donné ne doit-on en dens regarder. (Proverbes ruraux et vulgaux, $\mathrm{XIII}^{\mathrm{e}}$ )

21) Al verano que avía grano, que ya no pía Gonçalo. (Hernán Núñez, $\mathrm{XVI}^{\mathrm{e}}$ )

22) Al thyng which that shineth as the gold nis nat gold. (Chaucer, fin $\mathrm{XIV}^{\mathrm{e}}$ )

Je doute fort qu'on puisse les remettre aisément en circulation. C'est que contrairement à une vulgate fortement établie, les proverbes se modifient au cours du temps, voient leur syntaxe et leur morphologie révisées, leur lexique mis à jour, etc. Certains disparaissent parce que leur contenu ne correspond plus à l'actualité (Le saint de la ville n'est 
pas adoré (La Vëprie, Proverbes communs, 1495), «Nul n'est prophète en son pays»), d'autres sont remplacés parce que trop archaïsants (A buen vino, no ha menester pregonero $\rightarrow$ El buen paño, en el arca se vende). Enfin, il convient d'être méfiant, car certains changent de statut: ainsi $A$ bon entendeur, salut est une phrase situationnelle, après avoir été phrase parémique jusqu'à la moitié du XIX ${ }^{\mathrm{e}}$ siècle ${ }^{6}$. Parmi les transformations que peut subir un proverbe au cours de son histoire, la plus fréquente est la troncature (étudiée en détail dans Oddo, 2012). Par exemple En todas partes cuecen habas provient d'un En todas partes cuecen habas, y en mi casa a calderadas. Il arrive que cette troncature fasse passer d'une phrase parémique à une phrase situationnelle, ainsi : Fais ce que dois, advienne que pourra $\rightarrow$ Advienne que pourra ${ }^{7}$, Algo es algo, dijo al ver el hueso el galgo $\rightarrow$ Algo es algo, etc.

Si donc la diachronie peut fournir des indices, elle ne fournit pas de preuves absolues.

\subsection{Les indices traductologiques}

Une autre piste à explorer consiste à examiner les équivalents d'une phrase parémique dans les langues proches. En effet, ce qui porte une généricité non explicite dans une langue peut présenter des marques explicites dans une autre. Prenons le cas de Chien qui aboie ne mord pas: l'absence d'article peut laisser perplexe quant à la généricité du tout. On remarque cependant que l'équivalent anglais $A$ barking dog never bites comprend un indéfini à lecture générique, agrémenté d'un adverbe aspectuel itératif. Là encore, il convient d'être prudent: certes, il existe effectivement un fonds commun important issu de la culture latine: par exemple Une hirondelle ne fait pas le printemps a des équivalents proches dans beaucoup de langues, sur la base d'un modèle latin classique Hirundo una non effecit ver. On ne peut cependant généraliser: la phrase sentencieuse $A$ bon entendeur, salut vient d'un modèle latin Intelligenti pauca (Plaute), qui a donné $A$ buen

6 Alors que sur la même base latine, l'espagnol a forgé $A$ buen entendedor, pocas palabras bastan, qui est resté phrase parémique.

7 Notons que Fais ce que dois, advienne que pourra ne pourrait que difficilement être remis en circulation, sa syntaxe étant par trop déviante. Si Advienne que pourra a pu se maintenir comme phrase situationnelle, c'est en tant qu'expression figée (et seule de son espèce, sauf erreur de ma part). 
entendedor, pocas palabras (espagnol) et $A$ word to the wise is enough (anglais). Mais les versions anglaise et espagnole sont des phrases parémiques, alors que la leçon française est une phrase situationnelle.

\subsection{Les matrices rythmiques}

Comme je l'ai rappelé dans plusieurs publications dès Anscombre (1999: 26), il a été très tôt remarqué que les phrases parémiques présentaient parfois des assonancements, allitérations, effets de rimes, etc. Ainsi Anscombre (2012b: 147):

Comme lont signalé tous les parémiologues, létude des phrases parémiques fait apparaître des assonances, allitérations et répétitions. Morawski (1925) parle ainsi des particularités métriques des proverbes français antérieurs au $\mathrm{xv}^{\mathrm{e}}$ siècle, et affirme que cette métrique reste cependant pauvre, et limitée à des distiques isosyllabiques pour la plupart. Mais il s'empresse d'ajouter qu’on rencontre fréquemment des vers inégaux, ainsi que des schémas tripartites soit isorimiques, soit à rime orpheline, ou simplement marqués par l'allitération ou la cadence $^{8}$, phénomènes qui pour lui "ont presque toujours une origine littéraire certaine» (p. XIX). Nous avons contesté ici-même cette dernière affirmation. Les parémies ont très largement circulé entre peuple et écrivains, et de tels cycles excluent par nature le problème de l'origine. Il s'agit au fond d'une vieille hypothèse que nous avons combattue: le peuple serait incapable d'une invention poétique et en particulier rimique en fait réservée aux lettrés.

Nous proposerons une autre hypothèse, à l'appui de laquelle nous invoquerons l'examen de détail - jamais mené à notre connaissance ${ }^{9}$ - de ces phénomènes d'allitérations, d'assonances et répétitions.

Cette thèse que je soutiens n'est pas celle d'allitérations ou d'assonancements, mais en fait la suivante: les parémies - ou du moins certaines d'entre elles - sont en fait des occurrences de certaines configurations rythmiques. Je rejoins ainsi une thèse esquissée déjà dans Dessons (1984), qui voit dans les proverbes des schémas prosodiques. Quelles sont ces structures? Une hypothèse tentante - mais qui

8 Cette thèse du distique isosyllabique et/ou avec rime est en particulier reprise par Greimas (1970), Milner (1969), Rodegem (1972), parmi beaucoup d'autres.

9 Exception faite de certains travaux de Rodegem. 
reste à vérifier dans le détail - serait que ces schémas rythmiques sont en nombre restreint, et représenteraient une sorte de "poésie» naturelle propre à la langue, de la même façon qu'il y a en langue une astronomie «naturelle», ou une biologie «naturelle». Et de cette poésie naturelle serait née la poésie tout court, après une lente maturation ${ }^{10}$. Thèse inverse d'une vulgate répandue selon laquelle ces structures rythmiques proviendraient d'une poétique au sens noble dont elles ne seraient qu'une forme abâtardie (Anscombre, 2012b: 152 sq.).

En d'autres termes, les matrices rythmiques parémiques sont les mêmes que celles de la poésie tout court. En voici un exemple, celui de Cuando las barbas/de tu vecino/veas pelar/echa las tuyas/a remojar, de structure $a(5) b(5) a(5) c(5) a(5)$, à savoir ce que l'on appelle un romancillo en métrique espagnole ${ }^{11}$.

Cette remarque est suffisante en soi pour montrer que l'identification des parémies ne pourra pas se faire sur la seule présence d'une matrice rythmique. En effet, d'autres textes que les phrases parémiques exhibent les mêmes matrices rythmiques. Tout d'abord les vers des poésies habituelles connues, qui peuvent d'ailleurs aboutir à des phrases parémiques (L'ennui naquit un jour de l'uniformité) ou non (Caminante no hay camino). Mais aussi les comptines (Chocolate, molinillo, corre, corre, que te pillo/Croix de bois, croix de fer, si je mens je vais en enfer, etc.) ainsi que certains slogans, comme: Omo est là et la saleté s'en va/Pescados de crianza, pescados de confianza. Par ailleurs, les matrices rythmiques ne sont pas typiques des phrases parémiques, mais du cour de cette catégorie. Elles en sont le prototype, mais il n'est pas nécessaire d'exhiber une des structures rythmiques pour être une phrase parémique. Ainsi: Las apariencias engañan ou Chacun voit midi à sa porte. En fait, les matrices rythmiques donnent lieu à des cycles, comme le suivant: Las paredes han oýdos (Celestina, 1499) ${ }^{12} \rightarrow$ Las paredes han oýdos (Garay, 1541; Vallés, 1549); En consejas, las paredes han orejas (Vallés, 1549; Núñez, 1555; a(4) b(4) a(4)) $\rightarrow$ Las paredes han

10 C'est déjà l'avis de Fray Martín Sarmiento, qui déclare au XviII ${ }^{\mathrm{e}}$ siècle: «Han sido los refranes y adagios los que dieron base a las formas poéticas de España» (ce sont les proverbes et les adages qui ont fourni la base des formes poétiques de l'espagnol).

11 En fait, le romancillo authentique rime sur les vers pairs, et non comme ici sur les vers impairs.

12 À partir du latin médiéval Parietes habent aures 'les murs ont des oreilles'. 
oídos y los montes ojos (Correas, ca. 1630; a(8) b(6)); En consejas, las paredes han orejas (Correas, ca. 1630; a(4), b(4), a(4))) $\rightarrow$ En consejas, las paredes han orejas (Cejador y Frauca, 1929); Las paredes han oídos $y$ los montes ojos (Cejador y Frauca, 1929; a(8) b(6)) $\rightarrow$ En consejas, las paredes han orejas (Bergua, 1945; Ac., 1956) $\rightarrow$ Las paredes oyen (commun), pour ne citer que les principales étapes. Ce cycle est assez caractéristique: à partir d'une phrase parémique n'exhibant aucune matrice rythmique (Las paredes han oýdos), apparaissent une ou plusieurs variantes avec cette fois une matrice rythmique (En consejas, las paredes han orejas + Las paredes han oídos y los montes ojos). Généralement, l'une des variantes supplante les autres (En consejas, las paredes han orejas), puis divers facteurs ${ }^{13}$ peuvent faire que la parémie perde sa matrice rythmique (Las paredes oyen). La conformité à une matrice rythmique n'est donc pas permanente: elle peut être acquise ou perdue, selon l'endroit du cycle où l'on se trouve.

Je voudrais ajouter un argument supplémentaire en faveur du fait que les matrices rythmiques des parémies, même si ces combinaisons sont conformes à des schémas poétiques, ne suffisent pas à assurer le statut parémique. On peut en effet remarquer que les schémas rythmiques des phrases parémiques correspondent toujours à un lien sémantique. Ce peut être soit un lien de cause à conséquence: $A$ la Chandeleur/l'hiver trépasse/ou prend rigueur (a(5) b(4) a(4)) ${ }^{14}$, Agua del cielo/no quita riego (a(5) a(5)); soit de la même idée (générique) exprimée de deux façons différentes: ainsi pour l'espagnol Cada loco/ con su tema/y cada llaga/con su postema $(\mathrm{a}(4) \mathrm{b}(4) \mathrm{c}(5) \mathrm{a}(5))$, ou pour le français, Eil pour oil/dent pour dent (a(3), b(3)). En revanche, lorsque les phrases situationnelles exhibent des schémas rythmiques, un tel lien sémantique est généralement absent. En voici quelques exemples: A otra cosa, mariposa (a(4) a(4)), Tanto monta, monta tanto, Isabel como Fernando $(\mathrm{a}(4), \mathrm{b}(4) \mathrm{c}(4) \mathrm{a}(5))$; C'est bonnet blanc et blanc bonnet $(\mathrm{a}(4) \mathrm{b}(4)$, plus des assonancements). De ce point de vue, les structures rythmiques des phrases situationnelles s'apparentent aux internal rhymes comme Simer, Albert (fr.); En fin, Serafin (esp.); Let's

13 Dans le cas présent, il s'agit de facteurs linguistiques. Entre autres, haber n'est plus utilisé pour la possession, et la faculté auditive s'exprime plutôt au moyen de oído que de oreja.

14 Il s'agit là d'un schéma $\mathrm{a}(\mathrm{x}) \mathrm{b}(\mathrm{y}) \mathrm{a}(\mathrm{y})$ courant dans le domaine parémique. 
go, tally-ho! (angl.), etc. Pour ce qui est des comptines - qui présentent également des structures rythmiques "standards»- elles peuvent tirer soit du côté des «internal rhymes»: Colorín/colorado/Este cuento/Se ha acabado $(\mathrm{a}(4) \mathrm{b}(4) \mathrm{c}(4) \mathrm{b}(4))$; A la tienne/Étienne/à la tienne/mon vieux $(\mathrm{a}(3) \mathrm{a}(2) \mathrm{a}(3) \mathrm{b}(2))$. Soit du côté d'authentiques structures rythmiques: Promenons-nous dans les bois / quand le loup n'y est pas / si le loup y était/il nous mangerait $(\mathrm{a}(6) \mathrm{a}(6) \mathrm{b}(5) \mathrm{b}(5))$; Al sol le llaman Lorenzo / Y a la luna, Catalina/Cuando Lorenzo se acuesta/Se levanta Catalina $(\mathrm{a}(8) \mathrm{b}(8) \mathrm{a}(8) \mathrm{b}(8)$, en tenant compte des synalèphes).

\subsection{Les matrices lexicales}

Les matrices lexicales, appelées au départ schéma d'événements ${ }^{15}$, étaient destinées à proposer une alternative à une notion de figement qui devenait abusive. Il s'agit d'un concept qui concerne la production d'unités lexicales en tant qu'association d'une forme et d'un sens. Le premier phénomène étudié selon cette orientation théorique a été la formation $N_{1}$ à $N_{2}$, (Anscombre, 1990) ainsi moulin à vent, boîte à lettres, etc. $\grave{A}$ un «moule» fixe comprenant un mot grammatical constant à et des unités lexicales variables $N_{1}$ et $N_{2}$ était associé un invariant sémantique unique: $N_{1}$ et $N_{2}$ doivent être reliés par une phrase générique d'un type déterminé. C'est cet invariant sémantique qui permettait d'expliquer la possibilité de train à vapeur face à l'impossibilité de train à wagon-restaurant. C'est entre autres à travers l'étude des proverbes que m'est venue l'idée de généraliser cette notion de la façon suivante, reprise de (Anscombre, 2015: 27):

Une matrice lexicale sera un schéma comportant des unités linguistiques fixes et des variables linguistiques, et tel que:

a) les unités linguistiques fixes sont des éléments grammaticaux,

b) les variables linguistiques représentent des éléments lexicaux,

c) ce schéma est productif, au moins dans une certaine mesure,

d) les contraintes régissant l'instanciation des variables proviennent uniquement de la structure elle-même, sous forme d'un invariant sémantique.

15 J'ai par la suite renoncé à cette dénomination pour éviter une possible confusion avec les event patterns à la Parsons (1990). 
Il s'agit donc d'une théorie d'engendrement de nouvelles unités lexicales, et qui permet d'expliquer certaines de leurs propriétés sans avoir recours à la notion de figement. De plus, une matrice lexicale est la combinaison d'un invariant formel et d'un invariant sémantique: il s'agit donc d'une orientation qui a des points en commun avec la théorie Sens-Texte de Mel'čuk (v.g. 1995, 2011), et les Constructions de Goldberg (1995), qui naissent à peu près vers la même époque. Il est en revanche erroné d'y voir, comme l'affirme Marcon (2016) un emprunt à la notion de esquema fraseólogico de Zuluaga (1980:111) ${ }^{16}$. Outre que la notion de schéma d'événements ou matrice lexicale concerne la productivité morpho-sémantique, celle de Zuluaga d'une part se veut interne à une théorie du figement et d'autre part, concerne des structures purement syntaxiques, sans jamais évoquer d'invariant sémantique ${ }^{17}$. Or un tel invariant est primordial: Anscombre (2016b) propose une solution au fameux problème heureusement que $p /{ }^{*}$ malheureusement que $p$ à l'aide précisément de cette notion de matrice lexicale, et c'est essentiellement dans l'invariant sémantique que réside le nœud du problème plutôt que dans un quelconque problème de figement ${ }^{18}$. Quoi qu'il en soit, la notion de matrice lexicale éclaire celle de phrase parémique d'un jour nouveau. En effet, un certain nombre de phrases parémiques exhibent des invariants formels en petit nombre. Ce sont les phrases parémiques prototypiques - elles forment le cœur de la catégorie des phrases parémiques, et elles possèdent un sens compositionnel et un sens non directement déductible, le sens gnomique ou proverbial, parfois difficile à circonscrire. D'où l'idée

16 "[...] un esquema fraseológico constituye un molde sintáctico rígido cuya realización el hablar está sometida limitaciones de la libertad de combinación sólo, explicables mediante el concepto de fijación fraseológica. Se trata de algo así como unidades fraseológicas constituidas solamente por casillas libres y los elementos relacionales (gramaticales) fijos que las unen [...]». Zuluaga s'inspire de la notion de lexie complexe de Pottier (1968) et de celle molde sintáctico de Casares (1950). L'utilisation de la notion de schéma phraséologique que fait Zuluaga est critiquée dans Mura (2012: 89sq.).

17 L'erreur de Marcon provient de ce qu'il a omis l'invariant sémantique dans la définition qu'il évoque de matrice lexicale.

18 La solution consiste à voir cette construction comme issue d'une matrice lexicale Adverbe $+q u e+p$, le que étant non pas complétif, mais médiatif, et l'invariant sémantique attaché contraignant le type d'adverbe pouvant figurer dans cette matrice. 
que les phrases parémiques prototypiques sont produites par des matrices lexicales. On en dérive immédiatement que ce qui caractérise un proverbe est la superposition indissociable d'une forme et d'un sens. En conséquence de quoi, la reconnaissance du statut proverbial ne peut être uniquement un problème de forme. Ainsi, les phrases situationnelles, même si elles exhibent des schémas rythmiques et certaines formes spécifiques, ne seront pas perçues comme des phrases parémiques, faute de correspondre à une matrice lexicale: il y manquera toujours l'invariant sémantique. De même, les slogans (Qui resquille, sa propre poche il pille, SNCF) ou les jeux de mots comme Qui trop embrasse, rate son train, sont pris pour ce qu'ils sont - des slogans et des jeux de mots - et non pour des phrases parémiques, malgré leur adéquation à l'un des invariants formels des parémies. D'ailleurs, les tentatives de reconnaissance des phrases parémiques basées sur la seule forme donnent des résultats très décevants. En d'autres termes, on ne fera pas l'économie, pour l'analyse du phénomène parémique, de l'étude d'un sens qui a ceci de particulier qu'il est non compositionnel. Cet invariant sémantique attaché aux phrases parémiques repose sur un pattern déductif (Anscombre, 1984; Kleiber, 1989; Riegel, 1987), appelé selon les cas relation argumentative ou pivot implicatif, et un pattern déductif à valeur générique. C'est à mettre en évidence cette généricité et sa nature que va être consacré le reste de l'étude.

\section{Quelle généricité pour les phrases parémiques?}

\subsection{La généricité en question}

Tous les parémiologues sont d'accord sur ce point - une fois n'est pas coutume: les phrases parémiques, qu'elles soient proverbe, dicton, adage, tautologie, etc., ont en commun le caractère gnomique. Elles consignent une vérité générale, à prétention universelle: en bref, elles sont non événementielles, génériques/gnomiques. Si cette propriété est très largement acceptée, elle n'a en revanche jamais fait l'objet d'études particulières. Cette gnomicité/généricité touche au temps et à l'aspect, d'où l'idée d'examiner les rapports - dont nous verrons qu'ils sont particuliers - entre les phrases parémiques et l'aspect/le temps.

Un des problèmes que posent les phrases parémiques est leur hétérogénéité, qui rend difficile d'établir des règles générales faisant 
abstraction de la structure superficielle. On se trouve en effet face aux phénomènes suivants:

a) Certaines phrases situationnelles et certaines phrases parémiques ont des formes très proches, ce qui ne facilite pas l'identification de leur appartenance. Ainsi:

23) Il y a un Bon Dieu pour les ivrognes.

24) Il y a de l'eau dans le gaz.

Problème dû à l'ambiguïté de il y a en français, qui peut signifier à la fois la stativité 'il existe', où la résultativité «un événement fait qu'on a abouti à tel résultat».

Les phrases comportant des indices personnels peuvent cependant prêter lieu à interprétation gnomique:

25) Faites ce que je dis, ne faites pas ce que je fais.

26) Nunca digas de esta agua no beberé.

27) Arrieros somos, y en el camino nos encontraremos.

b) Les phrases nominales n'ont pas toujours un statut clair:

28) Juventud, divino tesoro.

29) À bon entendeur, salut.

30) Aussitôt dit, aussitôt fait.

c) Enfin, le français et l'espagnol possèdent un certain nombre de formes sapientiales au prétérit / passé composé ${ }^{19}$, ce qui pose le problème de leur accession à la gnomicité:

31) Quien fue a Sevilla perdió su silla.

32) Paris ne s'est pas fait en un jour.

33) Habló el buey y dijo mu.

34) No se tomó Zamora en una hora.

35) Dijo la sartén a la caldera...

36) L'ennui naquit un jour de l'uniformité.

19 L'examen de l'évolution diachronique des formes parémiques montre cependant que de telles formes tendent à disparaître au profit de formes au présent, en particulier en français. Ainsi, à côté de la leçon classique El que/Quien fue a Sevilla perdió su silla, est apparue une leçon plus récente cette fois au présent: El que/Quien va a Sevilla pierde su silla. 


\subsection{Phrases habituelles fréquentatives et habituelles dispositionnelles}

Pour des raisons qui apparaîtront plus tard, je commencerai par revenir à une distinction de Kleiber (1987), reprenant et prolongeant les travaux de Carlson (1979), à savoir celle entre phrase habituelle fréquentative et phrase habituelle dispositionnelle. Je (ré-)utiliserai les exemples (Anscombre, 2013):

37) Max joue du piano.

38) Max joue du piano avec un doigt.

Elles peuvent sembler sémantiquement très proches, mais il n'en est en fait rien. (38) est une phrase fréquentative, qui dénote une corrélation régulière entre deux événements, à savoir « jouer du piano » et « utiliser un seul doigt». (37), en revanche, est une phrase dispositionnelle, présentant une séquence d'actions comme une propriété du sujet. Une glose possible de (37) serait «Max est pianiste», impossible avec (38): « ${ }^{\star}$ Max est pianiste avec un doigt». Un certain nombre de propriétés linguistiques séparent les deux types, en particulier:

a) Les fréquentatives admettent la combinaison avec toujours, souvent et parfois, à l'inverse des dispositionnelles ${ }^{20}$ :

39) Max joue (souvent + parfois + toujours) (??du piano + du piano avec un doigt) ${ }^{21}$.

b) Les fréquentatives peuvent se combiner avec il arrive que et non les dispositionnelles:

40) Il arrive que Max joue (??du piano + du piano avec un doigt).

Bien sûr, Il arrive que Max joue du piano est possible, mais au sens événementiel, et non au sens dispositionnel Il arrive que Max soit pianiste $^{22}$.

20 Ou rarement, peu souvent, etc. en contexte négatif, ainsi: «[...] En matière fiscale plus encore que dans toute autre, les conseilleurs sont rarement les payeurs [...]» (Corpus Le Monde, 2008-2013).

21 Rappelons que Max joue du piano est à comprendre comme «Max est pianiste». "Max est (parfois + souvent + toujours) pianiste», n'est donc possible que dans le cas d'une lecture événementielle, et non pas dispositionnelle.

22 Ce curieux phénomène recoupe une opposition qui, à mon avis, traverse toute la langue: à savoir l'opposition potentiel/actuel et qui concerne en fait le mode de présentation de certaines entités. L'exemple le plus connu est celui de l'opposition sauveteur/sauveur (Benveniste, 1975). Benveniste remarque qu'entre autres différences, ces deux mots présentent le prédicat sauver sous deux aspects différents: 


\subsection{Les phrases parémiques comme phrases fréquentatives}

Si maintenant on examine les phrases génériques comme:

41) Les chats chassent les souris.

Et si l'on admet qu'elle caractérise les chats (il s'agit d'une phrase typifiante a priori), on remarque que leur généricité disparaît si on les combine avec parfois, souvent, toujours, il arrive que, et on passe alors d'une phrase dispositionnelle à une phrase fréquentative, ce que l'on peut voir sur le contraste:

42) ${ }^{\star}$ Les chats chassent les souris, mais la plupart du temps, ils dorment.

43) ?Les chats chassent (parfois + quelquefois + souvent) les souris/ ?Il arrive que les chats chassent les souris.

44) Les chats chassent (parfois + quelquefois + souvent) les souris, mais la plupart du temps, ils dorment.

45) Il arrive que les chats chassent les souris, mais la plupart du temps, ils dorment.

Par ailleurs, les sujets parlants ne voient pas Les chats chassent (parfois + quelquefois + souvent) les souris comme une variante de Les chats chassent les souris, et certains refusent même ces combinaisons. Enfin, elles semblent inexistantes dans les corpus que nous avons consultés. Il n'en est pas de même pour les phrases parémiques. Dans la mesure où la phrase parémique comporte un verbe combinable avec les adverbes ci-dessus, ces combinaisons semblent très naturelles, et sont d'ailleurs fréquentes dans les corpus:

46) Alors, ne te fie pas aux apparences, tu sais qu'elles sont souvent trompeuses. (Lévy, Sept jours pour une éternité, 2003)

47) Chassez le naturel, il revient toujours au galop, même devant une caméra de télévision. Le pari était risqué. Il se révèle pertinent. Par sa démarche, le réalisateur empêche le président

un sauveur n'est tel que s'il a effectivement sauvé quelqu'un, alors qu'un sauveteur est caractérisé par sa compétence à sauver, et peut parfaitement n'avoir jamais sauvé personne. L'action de sauver renvoie à une propriété potentielle dans sauveteur, et à une propriété actuelle dans sauveur. Cette opposition se retrouve dans les composés $N_{1}$ à $N_{2}$ : Anscombre (1990) remarque que l'opposition entre le type robe à fleurs et le type boîte à sel se ramène à précisément à actuel / potentiel : il y a effectivement des fleurs sur une robe à fleurs, mais même vide, une boîte à sel est destinée à contenir du sel. 
du Front national de se poser en victime, comme c'est souvent le cas sur les plateaux de télévision. (Le Monde, Corpus 2007-2013)

48) quand on a trouvé le bon, le vraiment bon endroit, il faut jeter l'ancre. Absolument. Qui va à la chasse peut perdre sa place, non? Je n'allais pas faire de promenade parce que je ne voulais pas manquer le thé de cinq heures, les conversations de n'importe quelle heure entre Babeth et Mona. (Forlani, Gouttière, 1989)

49) On n'a pas tort de dire: le mieux est parfois l'ennemi du bien. La sagesse sera de prévenir Ellynn le plus tard possible. (Mallet, Ellynn, 1985)

50) L'arbre cache toujours la forêt. Les fiançailles maudites de GDF et de Suez ne doivent pas faire oublier le grand Meccano énergétique qui se construit en Europe. (L'Express, 30/11/2006)

51) Il arrive que le hasard fasse bien les choses. Ainsi lorsque, au cours de quelques semaines, des ouvrages qui entretiennent des rapports parfois étroits, parfois secrets, se rassemblent sur la table du chroniqueur pour être divisés en deux piles. (Le Monde, 2007-2013)

En fait, une phrase comme Les chats chassent les souris et une phrase comme par exemple Les apparences sont trompeuses n'ont pas le même statut. La première représente un savoir objectif, fondé sur la «réalité» : d'où une certaine difficulté à minorer la généralité de cette réalité. Dans le cas des phrases parémiques en revanche, ce savoir n'est qu'un dire, qui peut fort bien être modulé par confrontation avec une réalité événementielle. Plusieurs propriétés vont dans ce sens:

a) L'opposition comme on sait/comme on dit:

52) (Comme on sait $+{ }^{*}$ comme on dit), les chats chassent les souris.

53) (Comme on sait + comme on dit), (Les apparences sont trompeuses + le hasard fait bien les choses...+$)$.

b) Les versions avec adverbes ne sont pas des variantes des phrases parémiques, comme on le voit sur les contrastes:

54) Comme on dit, les apparences sont trompeuses/?Comme on dit, les apparences sont parfois trompeuses.

55) Comme on dit, l'enfer est pavé de bonnes intentions/?Comme on dit, l'enfer est souvent pavé de bonnes intentions.

Notons par ailleurs que les phrases situationnelles sont événementielles, et n'admettent donc pas les adverbes d'itération: 
56) Nous sommes dans de beaux draps! ( ${ }^{\star}$ Les carottes sont parfois cuites + le mieux est l'ennemi du bien).

57) ${ }^{\star}$ Il a (parfois + souvent) coulé de l'eau sous les ponts depuis lors.

\subsection{Temps et phrases parémiques}

La différence essentielle entre les phrases parémiques et les phrases situationnelles réside en ce que les premières sont gnomiques et donc non événementielles, et que les secondes sont précisément événementielles. C'est ce qui fait qu'elles sont fréquemment accompagnées d'indications déictiques:

58) Je suis donc Luebig, un ex-haut fonctionnaire de la Gestapo... Depuis, beaucoup d'eau a coulé sous les ponts de Paris et d'ailleurs, n'est-ce pas? (San Antonio, Fais gaffe à tes os, 1956).

59) On ne pouvait même plus retourner chez Breton. Les flics allaient s'empresser de lui faire un brin de causette... Pour nous, les carottes étaient cuites. Dans quelques heures, Paris serait devenu une souricière. Plus question d'aller frapper aux portes. (Pécherot, Les mystères de la Butte, 2001)

60) La huida de estos jovencitos es mucho ruido y pocas nueces. En realidad, no huyen. Sólo buscan. Creen, inocentemente, que su escapada les dará lo que no han encontrado aquí. (Montserrat Roig, Tiempo de cerezas, 1976)

61) Aristóteles, prosiguió imperturbable, nunca se limitó a exponer lo que sucedía, sino que buscó el porqué... Lo que pasa es que desde entonces, ha llovido mucho. (Pérez-Reverte, El pintor de batallas, 2006)

Ce n'est bien sûr pas toujours le cas, et il convient donc d'établir d'autres critères. En voici un second, inspiré par un article de Deloor (2016), consacré au marqueur discursif comme quoi. Deloor établit que pour pouvoir dire comme quoi, $p$, $p$ doit nécessairement être une opinion que le locuteur estimait fondée antérieurement, dont il a pu douter, et dont un fait nouveau $q$ renforce pour lui le bien-fondé. Elle note de plus que dans les corpus qu'elle a consultés, $72 \%$ des $p$ sont des énoncés gnomiques. On peut comprendre pourquoi, dans la mesure où un énoncé gnomique peut très facilement être présenté comme un dire antérieur, rôle que les énoncés gnomiques jouent fréquemment, par exemple sous forme de présupposé (On sait qu'abondance de biens 
ne nuit pas), ou encore de cadre du discours (Étant donné que mieux vaut tenir que courir). Or il est tout à fait remarquable de constater que non seulement les énoncés gnomiques se combinent facilement avec comme quoi, mais aussi que cette combinaison est peu heureuse avec les phrases situationnelles ${ }^{23}$ :

62) ??Comme quoi, il a coulé de l'eau sous les ponts depuis lors.

63) ??Comme quoi, les carottes sont cuites.

64) ??Comme quoi, la confiance règne.

65) ${ }^{*}$ Comme quoi, un ange passe.

\section{Et le reste des phrases parémiques?}

\subsection{Les phrases parémiques nominales}

Tout ce que nous venons de voir possède l'inconvénient de ne s'appliquer qu'à des énoncés verbaux, i.e. possédant un verbe explicite. Or il existe des énoncés nominaux, et parmi ces énoncés nominaux, des phrases sentencieuses nominales, tant parémiques (De tal palo, tal astilla) que situationnelles (Beaucoup de bruit pour rien). Deux critères sont en fait à notre disposition, l'un aspectuel, et ayant trait à la gnomicité, et l'autre temporel, mettant en jeu l'antériorité temporelle du savoir représenté.

Le premier sera inspiré par une remarque de (Kleiber, 1983), qui note que les phrases génériques typifiantes a priori comme Les Alsaciens boivent de la bière se combinent avec des adverbes comme en général, qui indiquent une certaine généricité24:

66) En général, les Alsaciens boivent de la bière.

67) En général, les livres de linguistique sont chers.

68) En général, les chats chassent les souris.

Parmi de tels adverbes figure normalement, qui présente la réalisation d'un événement comme conforme à un ordre préétabli. Par exemple:

69) Normalement, le train pour Séville part à $13 \mathrm{~h}$.

23 Sauf, bien sûr, si la phrase situationnelle est présentée comme ayant déjà été dite. Ainsi: Comme quoi, il y a bien anguille sous roche.

24 Ce critère présente cependant un inconvénient, qui est que généralement y est ambigu entre Il est généralement vrai et On dit généralement. 
Or précisément, premier critère, les phrases parémiques nominales se combinent très bien avec normalement ${ }^{25}$ :

70) Normalement, tel père, tel fils.

71) Normalement, à coeur vaillant rien d'impossible.

72) Normalement, chose promise, chose due.

Le second critère sera celui-ci-dessus de la combinaison avec comme quoi :

73) Comme quoi, tel père, tel fils.

74) Comme quoi, à cour vaillant rien d'impossible.

75) Comme quoi, à malin, malin et demi.

L'inconvénient de ce dernier critère est qu'il est limité aux valeurs assertives, et convient mal ailleurs. Ainsi:

76) Comme quoi, (il ne faut pas se fier aux apparences +??ne te fie pas aux apparences).

77) Comme quoi, (il faut tenir ses promesses +??chose promise, chose due).

\subsection{Les exempla}

Il s'agit de phrases au passé composé ou au passé simple, et donc à un temps événementiel. Elles sont peu nombreuses, tant en français qu'en espagnol, et tendent à disparaître, nous l'avons dit. Elles fonctionnent sur le modèle de l'exemplum, étudié par Bizzarri et Rohde, et Darbord (cf. bibliographie): un événement particulier qui au départ, sert à illustrer une loi générale, finit par signifier cette loi générale. Or aucun des critères que nous avons évoqués plus haut ne s'applique de façon satisfaisante. Ainsi, le test de comme quoi:

78) ?Comme quoi, Paris ne s'est pas fait en un jour.

79) ??Comme quoi, l'ennui naquit un jour de l'uniformité.

80) ??Comme quoi, tel est pris qui croyait prendre.

et la seule combinaison faisant ressortir leur caractère de parole sapientiale semble être la suivante:

25 Comme me le fait remarquer à juste titre un des évaluateurs, la combinaison est meilleure avec en principe: En principe, tel père, tel fils/En principe, à cour vaillant rien d'impossible/En principe, chose promise, chose due. 
81) On a bien raison de dire que Paris ne s'est pas fait en un jour.

82) On a bien raison de dire que l'ennui naquit un jour de l'uniformité.

83) On a bien raison de dire que tel est pris qui croyait prendre.

On note lors que la combinaison avec comme quoi redevient possible:

84) Comme quoi, on a bien raison de dire que Paris ne s'est pas fait en un jour.

85) Comme quoi, on a bien raison de dire que l'ennui naquit un jour de l'uniformité.

86) Comme quoi, on a bien raison de dire que tel est pris qui croyait prendre.

Or dans une combinaison on a raison de dire que $p$, $p$ a le statut d'un présupposé, i.e. d'une énonciation virtuelle antérieure, ce qui explique la possibilité de comme quoi. On en déduit qu'apparemment, une assertion factuelle simple au passé (comme Paris ne s'est pas fait en un jour) n'est pas facilement interprétée comme une telle énonciation antérieure dont on ait pu douter.

Les exempla apparaissent donc vraiment comme une catégorie à part. Leur très petit nombre, et une totale absence de productivité plaident dans leur cas pour une hypothèse de figement ${ }^{26}$.

\section{Conclusion}

Cette petite étude - qui est loin d'avoir épuisé le sujet - permet cependant de tirer quelques conclusions utiles pour de futures études parémiologiques. La première est qu'on a largement sous-estimé le poids de la tradition grammaticale dans l'évaluation du fait parémique. On oublie trop souvent que cette grammaire - qui nous est si familière - n'est après tout qu'une théorie linguistique, avec donc des axiomes qu'on peut ne pas partager, surtout si on parvient à les falsifier, ce qui était le but recherché ici. Une seconde conclusion est l'intérêt que présente l'étude des formes sentencieuses, dont les nombreuses propriétés recoupent des phénomènes qui apparaissent dans d'autres

26 Ce qui ne signifie pas que toutes les phrases parémiques soient figées. Comme dans toutes les catégories lexicales, celle des phrases parémiques comporte des éléments figés, mais le figement n'est pas un trait caractéristique des phrases parémiques, comme je l'ai souvent exposé. Cf. par exemple Anscombre (2011). 
catégories concernées par le temps et l'aspect. Un dernier point enfin concerne le caractère trompeur de l'intuition quand elle est appliquée au champ parémique. En particulier en ce qui touche à la distinction entre parémiques et situationnelles, la frontière entre les deux catégories étant diachroniquement mouvante.

\section{Bibliographie}

ANSCOMBRE J.-C. (1990), Pourquoi un moulin à vent n'est pas un ventilateur, Langue française 86, 103-125.

ANSCOMBRE J.-C. (1999), Estructura métrica y función semántica de los refranes, Paremia 8, 25-36.

ANSCOMBRE J.-C. (2000), Parole proverbiale et structures métriques, Langages 139, 6-26.

ANSCOMBRE J.-C. (2006), Stéréotypes, gnomicité et polyphonie: la voix de son maître, in Perrin L. (éd.), Le sens et ses voix. Dialogisme et polyphonie en langue et en discours, Recherches linguistiques 28, Metz, Université de Metz, 349-378.

ANSCOMBRE J.-C. (2007), Hacia una clasificación lingüística de las formas sentenciosas, in Conde Tarrío G. (ed.), Nuevas aportaciones al estudio de las expresiones fijas, Fernelmont, E.M.E., col. Proximités, 11-37.

ANSCOMBRE J.-C. (2008), Quelques propriétés linguistiques des formes sentencieuses et leur application à la traduction francoespagnole, in González Royo C., Mogorrón Huerta P. (eds.), Estudios y análisis de fraseología contrastiva: lexicografía y traducción, Alicante, Facultad de Filosofía y Letras de la Universidad de Alicante, 11-36.

ANSCOMBRE J.-C. (2010), Las formas sentenciosas: un fenómeno lingüístico, Revista de Investigación Lingüística 13, 13-38.

ANSCOMBRE J.-C. (2011), Figement, idiomaticité et matrices lexicales, in Anscombre J.-C. \& Mejri S. (éds), Études sur le figement: la parole entravée, Paris, Champion, 17-40.

ANSCOMBRE J.-C. (2012a), Pour une théorie linguistique du phénomène parémique, in Anscombre J.-C., Darbord B. \& Oddo A. (éds) La parole exemplaire, Paris, Armand Colin, col. Recherches, 21-39.

ANSCOMBRE J.-C. (2012b), Matrices rythmiques et parémies, in Anscombre J.-C., Darbord B. \& Oddo A. (éds) La parole exemplaire, Paris, Armand Colin, col. Recherches, 147-158. 
ANSCOMBRE J.-C. (2013), Paremias, normas de acción y estructura semántica, in Olza I. \& Manero Richard E. (eds.), Fraseopragmática, Berlin, Frank \& Timme, col. Romanistik, 109-126.

ANSCOMBRE J.-C. (2015a), Les routines formulaires: problèmes de définition et de classification, in Mogorrón Huerta P. y Navarro Domínguez F. (eds.), Fraseología, Didáctica y Traducción, Studien zur romanischen Spachwissenschaft und interkulturellen Kommunikation, Band 101, Berne, Peter Lang, 15-35.

ANSCOMBRE J.-C. (2015b), Les parémies: variantes, matrices lexicales et familles parémiques, in Berbinski S. (éd.), Figement et imaginaire linguistique - de la langue à la traduction, Bucureşti, Editura Universitătii din București, 19-36.

ANSCOMBRE J.-C. (2016a), Énoncés parémiques et autonomie, in Anscombre J.-C., Darbord B., Oddo A. \& García de Lucas C. (éds), La phrase autonome. Théorie et manifestations, Gramm-R, vol. 31, Berne, Peter Lang, 101-115.

ANSCOMBRE J.-C. (2016b), Les constructions en adverbe que $p$ en français. Essai de caractérisation sémantique d'une matrice lexicale productive, Cahiers de lexicologie 1: 108, 199-224.

BENVENISTE E. (1975), Noms d'agent et noms d'action en indoeuropéen, Paris, Maison Neuve.

BIZZARRI H. O. \& ROHDE M. (éds) (2009), Tradition des proverbes et des exempla dans l'Occident médiéval/Die Tradition des Sprichwörter und exempla im Mittelalter, Berlin, Walter de Gruyter.

CARLSON G.-N. (1979), Generics and Atemporal, When, Linguistics and Philosophy 3, 49-98.

CASARES SÁNCHEZ J. (1950), Introducción a la lexicografía moderna, Madrid, CSIC.

DARBORD B. (2005), Réflexion sur la technique de l'exemplum dans le Libro de buen amor: fazañas, fablillas, parlillas, proverbios non mintrosos, en collaboration avec L. Valle Videla, in Heusch C. (ed.), Libro de buen amor de Juan Ruiz, Archiprêtre de Hita, Paris, Ellipses, 99-113.

DELOOR S. (2016), Le connecteur comme quoi: une mise en scène complexe, in Petit G., Haillet P.P. \& Salvador X. L. (éds), La dénomination: lexique, discours, Paris, Champion, 203-219.

DESSONS G. (1984), Pour une rythmique du proverbe, La Licorne $125,22-33$. 
GOLDBERG A. (1995), A Construction Grammar Approach to Argument Structure, Chicago and London The University of Chicago press.

GÓMEZ-JORDANA S. (2003), Taxinomie des proverbes français et espagnols contemporains, Revue de sémantique et pragmatique 13, 69-97.

GÓMEZ-JORDANA S. (2012), Les moules proverbiaux en français contemporain, in Anscombre J.-C., Darbord B. \& Oddo A. (éds) La parole exemplaire, Paris, Armand Colin, Col. Recherches La parole exemplaire, 114-132.

GREIMAS A. (1970), Les proverbes et les dictons, Du Sens, Paris, Le Seuil, 309-314.

GROSS G. (1996), Les éléments figés en français, Paris, Ophrys.

GROSS M. (1988), Les limites de la phrase figée, Langages 90, 7-22.

JANSSEN T. (1997), Compositionality, in Van Bentheme J. \& Ter Meulen A. (eds.), Handbook of Logic and language, Amsterdam/ Cambridge, Elsevier, MIT Press, 417-473.

KLEIBER, G. (1983), Remarques sur la généricité et la spécificité, Le Français moderne 51: 1, 36-49.

KLEIBER G. (1987), Du côté de la référence verbale. Les phrases habituelles, Berne-Francfort-New-York-Paris, Peter Lang.

KLEIBER G. (1989), Sur la définition du proverbe, Recherches germaniques 2, 233-252.

KLEIBER G. (2000), Sur le sens des proverbes, Langages 139, 39-58.

LLAMAS POMBO E. (2016), Le proverbe comme unité graphique autonome dans les manuscrits médiévaux, in Anscombre J.-C., Darbord B., Oddo A. \& García de Lucas C. (éds), La phrase autonome. Théorie et manifestations, Gramm-R, Berne, Peter Lang, vol. 31, 33-43.

MARTIN P. (2016), Phrase autonome et intonation autonome, in Anscombre J.-C., Darbord B., Oddo A. \& García de Lucas C. (éds), La phrase autonome. Théorie et manifestations, Gramm-R, vol. 31, Peter Lang, Berne, 33-43.

MELC'UK I. (1995), Phrasemes in Language and Phraseology in Everaert M. \& Van der Linden E.-J. (eds.), Linguistics, Idioms: Structural and Psychological Perspectives, Erlbaum, Hillsdale, 167-232.

MEL'ČUK I. (2011), Phrasèmes dans un dictionnaire, in Anscombre J.-C. \& Mejri S. (éds), Études sur le figement: la parole entravée, Paris, Champion, 41-61. 
MILNER G. (1969), De l'armature des locutions proverbiales, essai de taxonomie sémantique, L'Homme, Juillet-Septembre, 49-70.

MORAWSKI J. (1925), Proverbes français antérieurs au $x V^{e}$ siècle, Paris, Champion.

MARCON M. (2016), Autonomie lexico-syntaxique des parémies et reconnaisance auto-matique sur corpus, in Anscombre J.-C., Darbord B., Oddo A. \& García de Lucas C. (éds), La phrase autonome. Théorie et manifestations, Gramm-R, vol. 31, Berne, Peter Lang, 159-177.

MURA A. (2012), La fraseología del desacuerdo: los esquemas fraseológicos en español y en italiano, Tesis doctoral, Facultad de Filología, UCM, Madrid.

ODDO A. (2012), Phénomènes de troncature, in Anscombre J.-C., Darbord B. \& Oddo A. (éds) La parole exemplaire, Paris, Armand Colin, col. Recherches, 133-146.

PARSONS T. (1990), Events in the Semantics of English. A Study in Subatomic Semantics, Cambridge (MA), MIT Press.

POTTIER B. (1968), Lingüística moderna y filología hispánica, Madrid, Gredos.

RIEGEL M. (1987), Qui dort, dîne, ou le pivot implicatif dans les énoncés parémiques, in Riegel M. \& Tamba I. (dir.), L'implication dans les langues naturelles et dans les langages artificiels, Paris, Klincksieck, 85-99.

RODEGEM François M. (1972), Un problème de terminologie: les locutions sentencieuses, Cahiers de l'Institut de Linguistique de Louvain I: 5, 678-703.

TAMBA I. (2000), Formules et dire proverbial, Langages 139, 110-118.

TAMBA I. (2012), Retour au Chez Georges et sa cuisine proverbiale, in de Saussure L., Borillo A. \& Vuillaume M. (éds), Grammaire, Lexique, référence, regards sur le sens, Hommages offerts à Georges Kleiber, Berne, Peter Lang, 247-266.

TAMBA I. (2014), Sémantique lexicale et phrastique. Le sens compositionnel revisité, Cahiers de lexicologie 2: 5, 99-116.

ZULUAGA OSPINA A. (1980), Introducción al estudio de las expresiones fijas, Francfort sur le Main, Peter Lang. 
JEAN-CLAUDE ANSCOMBRE

\section{Annexes}

Formes sentencieuses étrangères mentionnées ${ }^{27}$

\section{Espagnol}

[Desde entonces], ha llovido mucho = Il est passé de l'eau sous les ponts. A buen entendedor, pocas palabras [bastan] = À bon entendeur, salut. A buen vino, no ha menester pregonero = À bon vin, point d'enseigne. A otra cosa, mariposa $=$ On passe au suivant.

Agua del cielo no quita riego $=$ Qu'il pleuve n'empêche pas d'arroser. Alsolle llaman Lorenzo, Y a la luna, Catalina, Cuando Lorenzo se acuesta,

Se levanta Catalina=comptine très connue et intraduisible. Algo es algo, [dijo al ver el hueso el galgo] = Vaut mieux ça que rien. Arrieros somos, y en el camino nos encontraremos = On se retrouvera. Cada loco/con su tema [y cada llaga con su postema] = À chacun son truc. Caminante no hay camino = Il n'y a pas de chemin tout tracé (il s'agit à l'origine d'un poème de Machado).

Chocolate, molinillo, corre, corre, que te pillo=comptine très connue et intraduisible.

Colorín colorado, este cuento se ha acabado=comptine très connue et intraduisible.

Cría cuervos, y te sacarán los ojos = Faites du bien à un vilain, il vous fait dans la main.

Cuando el río suena, agua lleva = Il n'y a pas de fumée sans feu.

Cuando las barbas de tu vecino veas pela echa las tuyas a remojar = Les ennuis n'arrivent pas qu'aux autres.

De tal palo, tal astilla $=$ Tel père, tel fils.

Dijo la sartén a la caldera [...] = C'est l'hôpital qui se moque de la charité. El buen paño, en el arca se vende = A bon vin, point d'enseigne.

En todas partes cuecen habas, [y en mi casa a calderadas]=Les gens sont les mêmes partout.

Habló el buey y dijo $m u=$ C'est parler pour ne rien dire.

Juventud, divino tesoro = C'est beau d'être jeune (il s'agit à l'origine d'un poème de Rubén Darío)

La confianza da asco = La confiance est la mère de tous les abus.

Las apariencias engañan = Les apparences sont trompeuses.

Las cosas de palacio van despacio = La justice est lente.

Las paredes oyen/Las paredes han orejas/Las paredes han oídos = Les murs ont des oreilles.

Mucho ruido y pocas nueces $=$ Beaucoup de bruit pour rien .

27 Les crochets signalent l'existence d'un usage tronqué. 
No se tomó Zamora en una hora = Paris ne s'est pas fait en un jour.

Nunca digas de esta agua no beberé= Il ne faut jamais dire Fontaine, je ne boirai pas de ton eau.

Pescados de crianza, pescados de confianza= Poissons d'élevage, poissons de confiance (slogan récent).

Quien fue a Sevilla perdió su silla= Qui va à la chasse, perd sa place.

Tanto monta, monta tanto, [Isabel como Fernando] =C'est bonnet blanc et blanc bonnet.

$Y$ pelillos a la mar =Et on tire un trait sur le passé.

\section{Anglais}

A friend in need is a friend indeed =C'est dans le besoin qu'on reconnaît les amis.

A rolling stone gathers no moss $=$ Pierre qui roule n'amasse pas mousse. $A$ barking dog never bites $=$ Chien qui aboie ne mord pas.

$A$ word to the wise is enough $=\overline{\mathrm{A}}$ bon entendeur, salut.

Let's go, tally-ho! $=$ En voiture, Simone.

Many a mickle mak's a muckle = Les petits ruisseaux font les grandes rivières.

\section{Portugais}

Quem tudo quer, tudo perde $=$ On risque de tout perdre en voulant trop gagner.

Mais vale um pássaro na mão que dois voando= $\mathrm{Un}$ bon tiens vaut mieux que deux tu l'auras. 